\title{
Association of White Matter Hyperintensities with Low Serum 25-Hydroxyvitamin D Levels
}

\author{
J.M. Prager, C. Thomas, W.J. Ankenbrandt, J.R. Meyer, Y. Gao, A. Ragin, S. Sidharthan, R. Hutten, and Y.G. Wu
}

\begin{abstract}
BACKGROUND AND PURPOSE: Vitamin D deficiency is associated with cognitive impairment in the elderly and with increased white matter T2 hyperintensities in elderly debilitated patients. We investigated the relationship between serum vitamin D and brain MR findings in adult outpatients.
\end{abstract}

MATERIALS AND METHODS: Brain MR studies of 56 patients ages 30-69 years were selected when vitamin D level had been obtained within 90 days of the MRI. White matter T2 hyperintensities were characterized by size and location by two neuroradiologists. Manual volumetric analysis was assessed in patients more than 50 years of age.

RESULTS: The entire cohort showed a significant negative relationship between serum 25-hydroxyvitamin $D$ and the number of confluent juxtacortical white matter T2 hyperintensities $(P=.047)$. The cohort ages 50 years and older showed stronger correlation between confluent white matter T2 hyperintensities and serum 25-hydroxyvitamin $D$ in the juxtacortical region; number $(P=.015)$ and size of white matter T2 hyperintensities $(P=.048)$. Atrophy was not significantly related to serum 25 -hydroxyvitamin $D$ by radiologist visual analysis or by the bicaudate ratio.

CONCLUSIONS: We found a significant relationship between vitamin $\mathrm{D}$ and white matter $\mathrm{T} 2$ hyperintensities in independent adult outpatients, especially over the age of 50 years.

ABBREVIATIONS: Vit $\mathrm{D}=$ serum 25 -hydroxyvitamin $\mathrm{D} ; \mathrm{WMH}=$ white matter $\mathrm{T} 2$ hyperintensities

$\mathrm{T}$ here is accumulating scientific evidence that vitamin D supplements can be protective against some chronic diseases. ${ }^{1-6}$ The National Institutes of Health supports a large ongoing study to test for such effects. ${ }^{2}$ Investigators have begun to test for brain imaging findings that correlate with serum vitamin D levels. ${ }^{6-8}$

The January 2010 issue of Neurology contained 3 studies that evaluated hundreds of elderly subjects to test for a relationship between vitamin $\mathrm{D}$ status and dementia or cognitive impairment. ${ }^{4,8,9}$ The Annweiler and Buell studies evaluated women only and both women and men, respectively; both showed a significant relationship between low vitamin D and dementia or cognitive impairment. The Slinin report only evaluated men and showed a

Received September 12, 2013; accepted after revision October 18

From the Department of Radiology (J.M.P., W.J.A., J.R.M., R.H., Y.G.W.), NorthShore University Health System, Evanston, Illinois; Department of Endocrinology (C.T.) University of Chicago, Chicago, Illinois; Departments of Statistics (Y.G.) and Radiology (A.R.), Northwestern University, Evanston, Illinois; and Department of Radiology (S.S.), Monmouth Medical Center, Long Branch, New Jersey.

Please address correspondence to Jordan M. Prager, MD, Department of Radiology, NorthShore University Health System, 2650 Ridge Ave, Evanston, IL 60201;

e-mail: jprager@northshore.org

http://dx.doi.org/10.3174/ajnr.A3840 trend for a relationship between vitamin $\mathrm{D}$ and cognitive impairment. A later study by Annweiler et al, ${ }^{10}$ with male and female subjects, showed lower serum 25-hydroxyvitamin D (Vit D) in patients with mild cognitive impairment compared with cognitively healthy individuals. A prospective study with 6-year follow-up involving more than 800 elderly patients showed significant increased risk of cognitive decline in those with low Vit D compared with sufficient levels. ${ }^{11}$

Studies have begun to examine the relationship between Vit D and brain MR findings in mice and in humans with significant disease. In 2010, Fernandes de Abreu et $\mathrm{al}^{7}$ examined the offspring of maternal vitamin D deficient mice by MR. The mice had smaller ventricles at the age of 30 weeks, which normalized by 70 weeks. Hippocampal volume significantly decreased from weeks $30-70$. Young mice also showed learning deficits. ${ }^{7}$ The Buell study evaluated 318 dependent, elderly patients with a mean age of 73 years and found a significant negative relationship between Vit D and white matter T2 hyperintensities (WMH) volume. ${ }^{8}$ Weinstock-Guttman et $\mathrm{al}^{6}$ published a 2011 study examining the relationship between Vit D and brain MR in 193 patients with multiple sclerosis. Their patients had a mean age of 46 years. They 
did not find a significant relationship between Vit D and WMH. However, a more recent longitudinal study showed a significant correlation between Vit D and the subsequent development of lesions in patients with multiple sclerosis. ${ }^{12}$ The Buell and Weinstock-Guttman studies did not account for dietary vitamin D supplementation.

The purpose of this study was to determine if Vit D levels are associated with white matter abnormalities in an outpatient population by MR imaging.

\section{MATERIALS AND METHODS}

With the use of our hospital information system, we identified patients 30-69 years of age who had been tested for Vit D and also had a routine MR brain study within a 90-day period for our pilot study. From each decade of age we then selected the 7 patients with the highest and the 7 with the lowest Vit D levels for review. Vit D level, age, race (black or white), history of hypertension, diabetes, and use of dietary vitamin D supplementation were evaluated. All MR images were de-identified in compliance with Health Insurance Portability and Accountability Act regulations. Our institutional review board approved the study.

\section{Image Acquisition and Analysis}

MR imaging studies were all performed on 1.5T Siemens Espree and Avanto or GE LX2 and Excite magnets by use of standard institutional protocols and included T1 sagittal/axial, T2 axial,

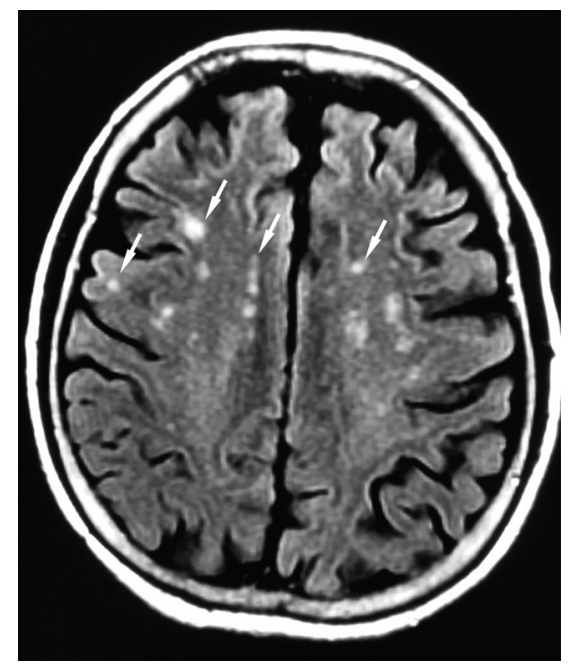

FIG 1. FLAIR image just above ventricles shows punctuate and confluent white matter T2 hyperintensities. From left to right: juxtacortical punctate, juxtacortical confluent, periventricular punctate, and central punctate.

Table 1: Demographics and clinical profiles

\begin{tabular}{|c|c|c|c|}
\hline Age, Years & $n$ & Vit $D<20$ & Vit $D \geq 20$ \\
\hline \multirow[t]{4}{*}{ 31-49; mean, 38.2} & 22 & 10 (7 female, 3 male) & 12 (11 female, 1 male) \\
\hline & & 7 white, 3 black & 12 white \\
\hline & & 1 hypertensive, 1 diabetic & 2 hypertensive, 2 diabetic \\
\hline & & 1 supplemental vitamin D & 8 supplemental vitamin D \\
\hline \multirow[t]{5}{*}{ 50-69; mean, 59.5} & 25 & 11 (7 female, 4 male) & 14 (11 female, 3 male) \\
\hline & & 7 white, 4 black & 13 white, 1 black \\
\hline & & 8 hypertensive (2 uncontrolled) & 10 hypertensive ( 2 uncontrolled) \\
\hline & & 4 diabetic (1 uncontrolled) & 3 diabetic (1 uncontrolled) \\
\hline & & 1 supplemental vitamin D & 7 supplemental vitamin D \\
\hline
\end{tabular}

FLAIR axial, and diffusion-weighted sequences. They were evaluated separately by 2 subspecialty-certified neuroradiologists, with more than 20 years (J.R.M.) and 16 years (W.J.A.) of experience interpreting brain imaging, for presence of infarction, atrophy (graded 1-4), and perivascular spaces. White matter T2 hyperintensities were evaluated for type: punctate, defined as $5 \mathrm{~mm}$ or less, versus confluent, greater than $5 \mathrm{~mm}$ in diameter; confluent lesions were further quantified by number and greatest diameter $(\mathrm{mm})$. White matter T2 hyperintensities were also evaluated for location; juxtacortical within $3 \mathrm{~mm}$ of cortex, periventricular within $3 \mathrm{~mm}$ of ventricle, or central (neither juxtacortical nor periventricular). When there was less than $7 \mathrm{~mm}$ between cortex and the ventricle, the distance was divided into thirds for localization.

Atrophy was further quantified by use of the bicaudate ratio, (minimum intercaudate distance divided by brain width along the same line), which reflects subcortical atrophy (Fig 1). ${ }^{13}$

MR imaging scans from the 25 participants more than 50 years of age were assessed for total WMH volume by use of MRIcro software (http://www.mccauslandcenter.sc.edu/mricro/mricro/ mricro.html). Rater W.J.A., an experienced neuroradiologist, performed manual volumetric measurement by use of axial 5-mm FLAIR images.

\section{Statistical Analysis}

We used Pearson and Spearman correlations to test for a significant relationship between MR findings and serum vitamin D levels. For analysis, we divided the patients into 3 groups: 1) the entire cohort; 2) those aged 50 and above; and 3) only those not taking supplements. The neuroradiologists' findings were evaluated for strength of agreement; their MR findings were evaluated separately and together. The punctate and confluent white matter changes were evaluated separately and in various combinations, for example, periventricular plus central plus juxtacortical punctate foci.

\section{RESULTS}

Patient profiles are shown in Table 1. Nine subjects were excluded because of incomplete MR imaging or confounding pathology (eg, brain tumor). Note the much higher incidence of black subjects in the low Vit D group and the marked increase in subjects taking dietary vitamin D supplementation in the higher Vit D group, 15 compared with 2 in the low Vit D group. There is also a larger ratio of women to men in the higher Vit D group, 22:4 compared with the low Vit D group, 14:7. Four of the 22 patients younger than 50 years of age had WMH compared with 20 of the 25 patients ages 50 and above. The incidence of hypertension (including uncontrolled, blood pressure $>140 / 90$ ) and diabetes (including uncontrolled, fasting glucose $>109$ ) was similar in both groups.

\section{Statistical Analysis}

Initial review by the combined readers of the entire cohort by use of Spearman correlation showed a significant nega- 


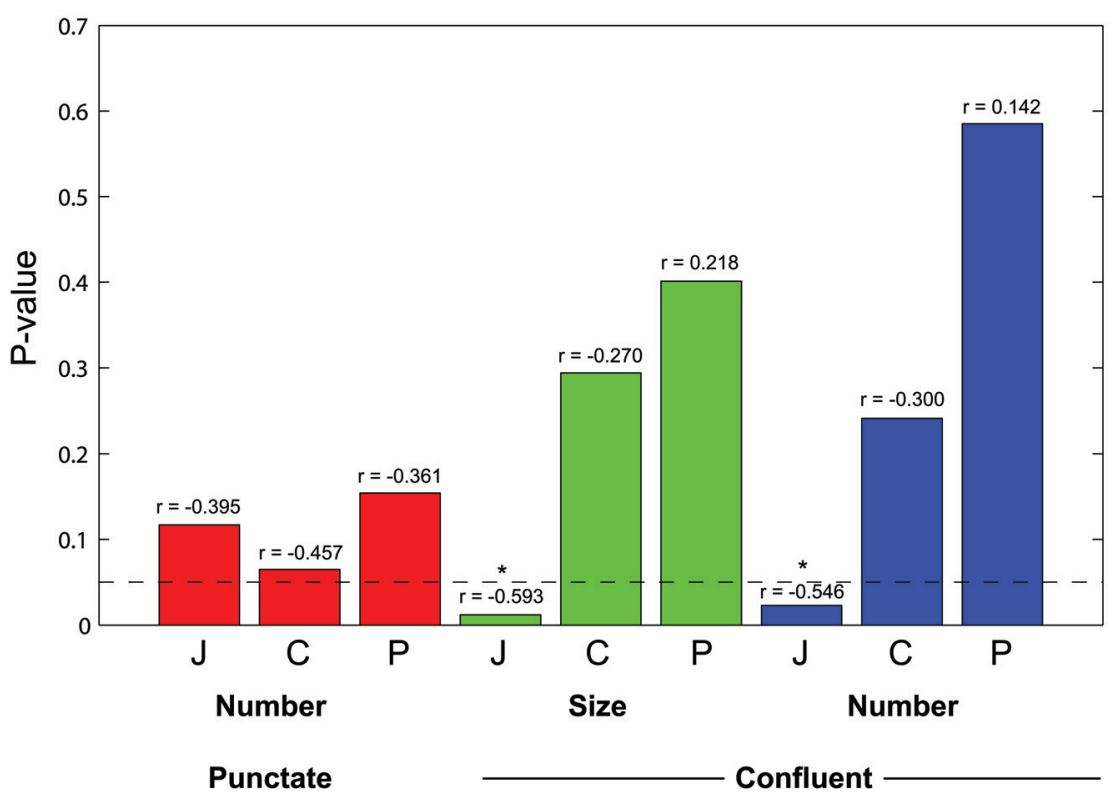

WMH Lesions

FIG 2. Probability value of correlation between Vitamin $D$ level and white matter $T 2$ hyperintensities in subjects without vitamin D supplementation by lesion location and type $(n=17$, age $>50$ years). Bar height represents $P$ value of relationship between $\mathrm{WMH}$ and serum 25 -hydroxyvitamin D. Bars lower than the horizontal line are less than .05 and are significant. Correlation coefficient $(r)$ value is shown above each bar. Red bars are punctate lesions, green bars are confluent lesions by size, and blue bars are confluent lesion number. J indicates juxtacortical; C, central; and P, periventricular.

Table 2: Pearson correlation of patients with migraine and WMH $(n=47)$

\begin{tabular}{llcc}
\hline & WMH Region & $\begin{array}{c}\text { Correlation } \\
\text { Coefficient }\end{array}$ & $\begin{array}{c}\boldsymbol{P} \\
\text { Value }\end{array}$ \\
\hline Punctate lesions & Juxtacortical & -0.283 & .054 \\
& Central & -0.409 & .004 \\
& Periventricular & -0.359 & .013 \\
Confluent lesions & Juxtacortical & -0.235 & .112 \\
& Central & -0.214 & .148 \\
& Periventricular & -0.321 & .028 \\
No. of lesions & Juxtacortical & -0.199 & .180 \\
& Central & -0.228 & .123 \\
& Periventricular & -0.301 & .040 \\
\hline
\end{tabular}

tive relationship between Vit D and the number of confluent juxtacortical WMH, $\rho=-0.292, P=.047$; reader 2 showed significant relationship with confluent juxtacortical WMH number, $\rho$ $-0.295, P=.044$ and size, $\rho=-0.298, P=.042$. Combined readers of the cohort age 50 years and above showed a significant correlation between confluent WMH and Vit D in the juxtacortical region (number of WMH, $\rho=-0.481, P=.015$; size of WMH, $\rho=-0.399, P=.048$ ). When subjects taking dietary vitamin D supplementation were excluded, there were 17 subjects 50 years of age and above. The combined readers showed significant relationship between Vit D and juxtacortical confluent WMH number $(\rho=-0.546 ; P=.023)$; and size $(\rho=-0.593$; $P=.012$ ) (Fig 2 ). In addition, reader 1 showed a relationship with central and combined central and periventricular punctate WMH ( $\rho=-0.490, P=.046$ and $\rho=-0.506, P=.038$, respectively); reader 2 showed a relationship with combined central and periventricular WMH $(\rho=-0.482, P=.05)$. There was no significant relationship between total WMH score and Vit D by vi- sual or manual volumetric analysis. There was no significant relationship in the cohort of those ages younger than 50 years. Atrophy was not significantly related to Vit D by radiologist evaluation or by the bicaudate ratio.

Patients with migraine showed strong relationships with punctate WMH (periventricular $P=.013$, central $P=.004)$. A significant relationship was also seen with size and number of confluent $\mathrm{WMH}$ in the periventricular region $(P=.028$ and .04 , respectively) (Table 2).

Significant relationships were identified between all location punctate $\mathrm{WMH}$ and hypertension (juxtacortical, $P=$ .023 ; central, $P=.001$; periventricular, $P=$.018). The number of juxtacortical confluent $\mathrm{WMH}$ was significantly related $(P=.049)$; periventricular confluent WMH were related by number and size $(P=.008$ and $P=.001$, respectively) (Table 3 ). The relationship between WMH and Vit D is attenuated when controlling for hypertension.

There was fair to moderate agreement between the 2 neuroradiologist evaluators. Interobserver $\kappa$ values ranged from $0.206-0.532$ except for central confluent lesion number when $\kappa$ value was 0.125 . The $P$ value for all WMH by type and location was $<.001$ except for central and juxtacortical maximum size confluent lesions when $P=.031$ and $P=.003$, respectively (Table 4 ).

\section{DISCUSSION}

We found a significant relationship between WMH and low Vit D in healthy outpatients ages 50 years and above. The relationship is stronger and more extensive when the patients taking supplemental vitamin D are excluded. We were further able to characterize the significant WMH as confluent in the juxtacortical white matter and punctate in the combined periventricular/central white matter.

The presence of WMH is concerning because of the known strong association with reduced cognition. ${ }^{14-19}$ The Austrian Stroke Prevention Study showed that baseline grading of WMH was a better predictor for progression of cognitive difficulties than age or the presence of hypertension. ${ }^{20}$ However, the presence of hypertension and diabetes are also associated with cognitive decline. ${ }^{21,22}$ The exact mechanism by which low vitamin D might be related to reduced cognition or the development of WMH is not known. It has been proposed that the cognitive dysfunction seen with low Vit D is more specifically caused by impaired mental shifting and information updating involving the dorsolateral circuit of the frontosubcortical neuronal circuit. ${ }^{23}$ Possible explanations may involve modulation of neurotrophins, calcium binding growth factors, and inflammatory cytokines. ${ }^{1}$ It has been suggested that Vit $\mathrm{D}$ has an important role in the differentiation and 
Table 3: Pearson correlations: WMH versus age, diabetes, and hypertension $(n=47)$

\begin{tabular}{|c|c|c|c|c|c|c|c|}
\hline & \multirow[b]{2}{*}{ WMH Region } & \multicolumn{2}{|c|}{ Age } & \multicolumn{2}{|c|}{ Diabetes } & \multicolumn{2}{|c|}{ Hypertension } \\
\hline & & $\begin{array}{l}\text { Correlation } \\
\text { Coefficient }\end{array}$ & $\begin{array}{c}P \\
\text { Value }\end{array}$ & $\begin{array}{l}\text { Correlation } \\
\text { Coefficient }\end{array}$ & $\begin{array}{c}P \\
\text { Value }\end{array}$ & $\begin{array}{l}\text { Correlation } \\
\text { Coefficient }\end{array}$ & $\begin{array}{c}P \\
\text { Value }\end{array}$ \\
\hline \multirow[t]{3}{*}{ Punctate lesions } & Juxtacortical & 0.364 & .012 & 0.129 & .389 & 0.331 & .023 \\
\hline & Central & 0.540 & 0 & 0.317 & .03 & 0.476 & .001 \\
\hline & Periventricular & 0.468 & .001 & 0.324 & .026 & 0.345 & .018 \\
\hline \multirow[t]{3}{*}{ Confluent lesions } & Juxtacortical & 0.277 & .06 & 0.297 & .042 & 0.274 & .062 \\
\hline & Central & 0.410 & .004 & 0.185 & .212 & 0.226 & .127 \\
\hline & Periventricular & 0.536 & 0 & 0.226 & .126 & 0.467 & .001 \\
\hline \multirow[t]{3}{*}{ No. of lesions } & Juxtacortical & 0.205 & .168 & 0.113 & .451 & 0.289 & .049 \\
\hline & Central & 0.353 & .015 & 0.269 & .067 & 0.263 & .074 \\
\hline & Periventricular & 0.471 & .001 & 0.232 & .117 & 0.385 & .008 \\
\hline
\end{tabular}

Table 4: Correlation between readers $(n=47)$

\begin{tabular}{lllc}
\hline & WMH Region & $\begin{array}{c}\boldsymbol{\kappa} \\
\text { Value }\end{array}$ & $\boldsymbol{P}$ Value \\
\hline Punctate lesions & Juxtacortical & 0.399 & $<.001$ \\
& Central & 0.394 & $<.001$ \\
& Periventricular & 0.215 & $<.001$ \\
Confluent lesions & Juxtacortical & 0.261 & $<.001$ \\
& Central & 0.125 & .031 \\
& Periventricular & 0.305 & $<.001$ \\
No. of lesions & Juxtacortical & 0.532 & $<.001$ \\
& Central & 0.206 & .003 \\
& Periventricular & 0.44 & $<.001$ \\
\hline
\end{tabular}

axonal adhesion of oligodendrogliocytes in the developing brain, with low Vit D increasing oligodendroglial apoptosis. This would prime an appropriate individual for the subsequent development of multiple sclerosis. ${ }^{24}$

Our findings concur with the 2 prior studies that investigated the relationship of Vit D and MR findings. The Buell study ${ }^{8}$ found a significant relationship between Vit D and WMH volume in older debilitated patients with average age of 73 years. Our patients are healthier and younger, with an average age of 59 years. This increases the range of patients in whom low Vit D should be considered in the differential of WMH. The Weinstock-Guttman study $^{6}$ evaluated patients with MS for a relationship with Vit D and WMH. They did not find a significant relationship, but their patients were younger, with an average age of 46 years. We also did not find a relationship in younger patients. Furthermore, having a higher baseline volume of WMH in patients with multiple sclerosis, a larger number of subjects would be necessary to establish a significant relationship with a small number of additional causes for WMH.

A more recent 5-year longitudinal study of relapsing MS evaluated changes in MRI and clinical status relative to vitamin D levels. The study included 469 patients with 2362 3T MR examinations; the average age was 42 years. They correlated baseline and subsequent vitamin D levels with the development of WMH, gadolinium enhancement, clinical disability, and relapses. There were significant reductions in new WMH, gadolinium-enhancing lesions, and disability correlating with each $10 \mathrm{ng} / \mathrm{mL}$ increase in vitamin $\mathrm{D}$ level. The clinical relapse rate was lower but not statistically significant. ${ }^{12}$

Our analysis is most limited by measurement of the vitamin D level, which represents a single point in a dynamic process. When one considers the clinical factors that influence the blood level of vitamin D and the clinical profile of WMH, it is apparent why statistical analysis needs to adjust for dietary vitamin D supplementation. Measured vitamin D changes with the season and with individual exposure to sunlight and it is stored in the body for up to 2 months. Vitamin D production decreases in darker skinned individuals and with age. ${ }^{3,25}$ The vitamin $\mathrm{D}$ level of an older patient taking a supplement does not reflect levels before taking supplements when WMH appeared. White matter T2 hyperintensities accumulate over a lifetime as they are only rarely reversible and then associated with acute illness such as Posterior Reversible Encephalopathy Syndrone or hepatic encephalopathy. Our patients probably had WMH years before they were examined with the use of MR and before they supplemented their diet with vitamin $\mathrm{D}$. We did not control the patient selection process regarding the reason for medical and imaging evaluation, which could lead to unforeseen bias.

Potentially problematic in our patient population is the presence of hypertension and diabetes mellitus, which are closely related confounders known to increase WMH. ${ }^{26-29}$ There is a similar incidence of controlled and uncontrolled hypertension (8 of $11,73 \% ; 10$ of $14,71 \%$ ) and diabetes ( 4 of $11,36 \% ; 3$ of $14,21 \%$ ) in the low and high Vit D groups.

This is a cross-sectional pilot study with a limited number of subjects and can only establish association but not causation. Future studies should consider including a larger cohort with more rigorous patient selection, especially patients who do not supplement their diet with vitamin D.

White matter T2 hyperintensities are a pathologic end point. Other MR techniques such as magnetization transfer, mean diffusivity, fractional anisotropy, perfusion, and MR spectroscopy have shown abnormalities in normal-appearing white matter in patients with $\mathrm{MS} .{ }^{30}$ Diffusion abnormalities are also seen in Alzheimer disease. ${ }^{31}$ Maillard et al, ${ }^{19}$ in a study of elderly subjects with cognitive health, cognitive impairment, and Alzheimer disease, showed an increased risk for white matter conversion to WMH in patients with lower fractional anisotropy and higher FLAIR signal in normal-appearing white matter. Optimized future studies should be longitudinal and use these MR techniques.

\section{CONCLUSIONS}

This study shows a previously unreported significant relationship between low Vit D levels and white matter injury evidenced by increased juxtacortical confluent WMH in healthy outpatients between the ages of 50-69 years. There was no relationship in our 
patients ages 31-49 years. We showed a more powerful relationship in patients who did not take vitamin D supplements because normal Vit D in such patients could disguise a history of chronic low Vit D before the onset of supplementation. This pilot study has significant limitations including a small number of subjects and patient selection factors. Longitudinal studies with a greater number and control of subjects and additional MR metrics are indicated to establish a more definitive relationship.

\section{ACKNOWLEDGMENTS}

The authors would like to thank Sally Gartman for her assistance with manuscript preparation and Dr. Robert Edelman for support and editorial assistance.

Disclosures: Joel Meyer—UNRELATED: Consultancy: Pfizer DMC Safety Committee for drug trial.

\section{REFERENCES}

1. Miller JW. Vitamin D and cognitive function in older adults: are we concerned about vitamin D-mentia? Neurology 2012;74:13-15

2. Brody J. Reasons that vitamin D may matter. The New York Times. March 13, 2012; D7

3. Holick MF. Vitamin D deficiency. N Engl J Med 2007;357:266-81

4. Annweiler C, Schott AM, Allali G, et al. Association of vitamin D deficiency with cognitive impairment in older women: cross-sectional study. Neurology 2010;74:27-32

5. Niino M. Vitamin D metabolites and multiple sclerosis. J Neurol Neurosurg Psychiatry 2011;82:121

6. Weinstock-Guttman B, Zivadinov R, Qu J, et al. Vitamin D metabolites are associated with clinical and MRI outcomes in multiple sclerosis patients. J Neurol Neurosurg Psychiatry 2011;82:189-95

7. Fernandes de Abreu DA, Nivet E, Baril N, et al. Developmental vitamin D deficiency alters learning in C57B1/6J mice. Behav Brain Res 2010;208:603-08

8. Buell JS, Dawson-Hughes B, Scott TM, et al. 25-Hydroxyvitamin D, dementia, and cerebrovascular pathology in elders receiving home services. Neurology 2010;74:18-26

9. Slinin Y, Paudel ML, Taylor BC, et al. 25-Hydroxyvitamin D levels and cognitive performance and decline in elderly men. Neurology 2010;74:33-41

10. Annweiler C, Fantino B, Schott AM, et al. Vitamin D insufficiency and mild cognitive impairment: cross-sectional association. Eur J Neurol 2012;19:1023-29

11. Llewellyn DJ, Lang IA, Langa KM, et al. Vitamin D and risk of cognitive decline in elderly persons. Arch Intern Med 2010 170:1135-41

12. Mowry E, Waubant E, McCulloch C, et al. Vitamin D status predicts new brain MRI activity in multiple sclerosis. Ann Neurol 2012;72: $234-40$

13. van den Heuvel DM, ten Dam VH, de Craen AJ, et al. Increase in periventricular white matter hyperintensities parallels decline in mental processing speed in a non-demented elderly population. J Neurol Neurosurg Psychiatry 2006;77:149-53

14. Yoshita M, Fletcher E, Harvey D, et al. Extent and distribution of white matter hyperintensities in normal aging, MCI, and AD. Neurology 2006;67:2192-98

15. Inzitari D, Simoni M, Pracucci G, et al. Risk of rapid global functional decline in elderly patients with severe cerebral age-related white matter changes: the LADIS study. Arch Intern Med 2007; 167:81-88

16. Gouw AA, van der Flier WM, Fazekas F, et al. Progression of white matter hyperintensities and incidence of new lacunes over a 3-year period: the Leukoaraiosis and Disability Study. Stroke 2008;39: $1414-20$

17. Debette S, Bombois S, Bruandet A, et al. Subcortical hyperintensities are associated with cognitive decline in patients with mild cognitive impairment. Stroke 2007;38:2924-30

18. Vannorsdall TD, Waldstein SR, Kraut M, et al. White matter abnormalities and cognition in a community sample. Arch Clin Neuropsychol 2009;24:209-17

19. Maillard P, Carmichael O, Harvey D, et al. FLAIR and diffusion MRI signals are independent predictors of white matter hyperintensities. AJNR Am J Neuroradiol 2013;34:54-61

20. Schmidt R, Enzinger C, Ropele $S$, et al. Progression of cerebral white matter lesions: 6-year results of the Austrian Stroke Prevention Study. Lancet 2003;361:2046-48

21. Knopman D, Boland LL, Mosley T, et al. Cardiovascular risk factors and cognitive decline in middle-aged adults. Neurology 2001;6:42-48

22. Akisaki T, Sakurai T, Takata T, et al. Cognitive dysfunction associates with white matter hyperintensities and subcortical atrophy on magnetic resonance imaging: Japanese Elderly Diabetes Intervention Trial (J-EDIT). Diabetes Metab Res Rev 2006;22:376-84

23. Annweiler C, Montero-Odasso M, Muir SW, et al. Vitamin D and brain imaging in the elderly: should we expect some lesions specifically related to hypovitaminosis D? Open Neuroimaging Journal 2012;6:16-18

24. Chaudhuri A. Why we should offer routine vitamine D supplementation in pregnancy and childhood to prevent multiple sclerosis. Med Hypotheses 2005;64:608-18

25. Rajakumar K, Greenspan SL, Thomas SB, et al. Solar ultraviolet radiation and vitamin D: a historical perspective. Am J Public Health 2007;97:1746-54

26. Murray AK, Staff RT, Shenkin SD, et al. Brain white matter hyperintensities: relative importance of vascular risk factors in nondemented elderly people. Neurology 2005;237:251-57

27. Swan GE, DeCarli C, Miller BL, et al. Association of midlife blood pressure to late-life congnitive decline and brain morphology. $\mathrm{Neu}$ rology 1998;51:986-93

28. Valdés Hernández MC, Piper RJ, Bastin ME, et al. Morphologic, distributional, volumetric, and intensity characterization of ventricular hyperintensities. AJNR Am J Neuroradiol 2014;35:55-62

29. Knopman DS, Penman AD, Catellier DJ, et al. Vascular risk factors and longitudinal changes on brain MRI. Neurology 2011;76: 1879-85

30. Filippi M, Rocca MA. MR imaging of multiple sclerosis. Radiology 2011;259:659-81

31. Stahl R, Dietrich O, Teipel SJ, et al. White matter damage in Alzheimer disease and mild cognitive impairment: assessment with diffusion-tensor MR imaging and parallel imaging techniques. Radiology 2007;243:483-92 